\title{
Primary Hepatic Lymphoma Resected by ALPPS Procedure (Associating Liver Partition and Portal Vein Ligation for Staged Hepatectomy)
}

\author{
Sorin Alexandrescu ${ }^{1,2}$, Luminita Stoica ${ }^{1}$, Razvan Grigorie ${ }^{1}$, Dana Tomescu ${ }^{2,3}$, Camelia Dobrea ${ }^{2,4}$, \\ Irinel Popescu ${ }^{1,2}$, Doina Hrehoret ${ }^{1}$
}

'Dan Setlacec Center of General Surgery and Liver Transplantation, Fundeni Clinical Institute, Bucharest, Romania

${ }^{2}$ Carol Davila University of Medicine and Pharmacy, Bucharest, Romania

${ }^{3}$ Carol Davila University of Medicine and Pharmacy, Department of Anaesthesia and Intensive

Care III, Fundeni Clinical Institute, Bucharest, Romania

${ }^{4}$ Department of Hematology, Fundeni Clinical Institute, Bucharest, Romania

\author{
Corresponding author: \\ Doina Hrehoret, MD \\ Dan Setlacec Center of General Surgery \\ and Liver Transplantation, \\ Fundeni Clinical Institute \\ Soseaua Fundeni, 258, Sector 2, \\ Bucharest, 022328, Romania \\ E-mail: doinitahrehoret@yahoo.com
}

\section{ABSTRACT}

Background: Primary hepatic lymphoma represents a very rare location of non-Hodgkin lymphomas, whose treatment consists of liver tumor resection followed by chemotherapy. In some cases, however, upfront hepatectomy is not feasible due to insufficient volume of the liver remnant.

Case report: An ALPPS (Associating Liver Partition and Portal Vein Ligation for Staged Hepatectomy) procedure was performed in a patient with a large primary hepatic lymphoma, whose estimated liver remnant represented only $13 \%$ of total liver volume, it was performed. During the first stage, we performed right portal branch ligation and in situ splitting, without right bile duct ligation. After the first stage, the patient did not develop biliary fistula, ascites or sepsis. The volume of segments 2 and 3 increased by $98 \%$ after eleven days, making possible subsequent resection of the tumor by a right trisectionectomy. The patient did not develop any complication, being discharged 9 days after the second stage of ALPPS. Postoperatively, the patient underwent chemotherapy, being disease-free at 13 months following operation.

Conclusion: This is the first patient presented in the literature who underwent ALPPS for resection of a primary hepatic lymphoma. This type of tumor could be managed by ALPPS, with low morbidity and mortality rates.

Abbreviations: ALPPS - Associating Liver Partition and Portal Vein Ligation for Staged Hepatectomy, PHL - primary hepatic lymphoma, PVE - portal vein embolization, PVL - portal vein ligation, FLR - future liver remnant

Key words: ALPPS, major liver resection, portal vein embolization, portal vein ligation, "twostage" liver resection, primary hepatic lymphoma

\section{INTRODUCTION}

Primary hepatic lymphoma $(\mathrm{PHL})$ is a non-Hodgkin lymphoma $(\mathrm{NHL})$ that is typically confined to the liver, without evidence of lymph nodes, spleen, 
hematogenous bone marrow or other lymphoid structures involvement (1). The optimal therapy is not fully established, although most cases reported to date were treated with combined chemotherapy (2). However, over the last decade, several reports suggested that liver resection associated with postoperative chemotherapy appears to improve survival when compared to chemotherapy alone (3-7).

Thus, in PHL patients, the only contraindication for liver resection is technical impossibility to perform a complete tumor resection. This situation is similar to those encountered in patients with other malignant liver tumors (e.g., hepatocellular carcinoma, intrahepatic cholangiocarcinoma, colorectal liver metastases) without extrahepatic metastases. In such instances, the most frequent clinical scenario that precludes liver resection is insufficient volume of the remnant liver parenchyma (future liver remnant - FLR), which should represent at least $25 \%$ of total liver volume (TLV) in order to avoid postoperative liver failure. For these patients, several strategies were developed: liver resection after portal vein embolization (PVE) or ligation (PVL), "two-stage" liver resection (associated or not with $P V L / P V E)$, resection after tumor shrinkage induced by preoperative chemotherapy, ultrasound-guided liver resections or combined ablation and resection (CARe).

Each of the above mentioned strategies presents some drawbacks: 1 . in patients scheduled for liver resection after PVE/PVL, the increase of the FLR volume could be insufficient and, moreover, some patients could develop metastases in the FLR during the interval between PVL/PVE and the time of hepatectomy, making this procedure futile in up to $50 \%$ of cases; 2 . patients scheduled for "two-stage" liver resection could develop new metastases in the FLR during the period between the two stages; 3 . tumor shrinkage could be insufficient to afford subsequent complete tumor resection in more than $75 \%$ of patients; 4 . ultrasoundguided liver resection is not operational for large tumors involving both right and left hemiliver; 5 . the long-term results achieved by ablation (even associated with resection - CARe) are significantly poorer than those achieved by complete tumor resection. To overcome these drawbacks, a few years ago, a new surgical procedure was developed in order to achieve a more robust hypertrophy of the FLR, in a shorter period of time. This procedure, called "associating liver partition and portal vein ligation for staged hepatectomy ALPPS", enables, at least theoretically, a higher increase of the FLR volume than PVE/PVL in a shorter period of time, decreasing the risk of development of new metastases in the FLR.
In the present manuscript, we report our experience with the first patient who underwent ALPPS at "Dan Setlacec" Center of General Surgery and Liver Transplantation from Fundeni Clinical Institute, Bucharest. We report short-term results in terms of FLR gaining and time of liver hypertrophy, as well as immediate and one-year postoperative course. Also, to the best of our knowledge, this is the first report of a PHL patient successfully addressed by ALLPS.

\section{CASE REPORT}

A 54 year-old woman with a BMI of 26.3 was initially seen for right upper quadrant tenderness. Of note, in 1998 she underwent total hysterectomy with bilateral anexectomy followed by radio-chemotherapy probably for carcinoma of the uterus (medical records unavailable). Her past medical history also included hydrocephalus (diagnosed in 2013), controlled by Acetazolamide.

Abdominal ultrasound revealed a large tumor involving right hemiliver, and a contrast-enhanced CT scan confirmed the presence of a tumor involving segments 4, 5, 6 and 8 .

The volumetric evaluation of the liver showed that the TLV was 2139 cubic $\mathrm{cm}(\mathrm{cmc})$, and the volume of segments $2-3$ was $294 \mathrm{cmc}$ (13.7\% of TLV - fig. 1). The radiologic features of the lesion were not suggestive of either hepatocellular carcinoma or intrahepatic cholangiocarcinoma, or of a benign liver tumor (hemangioma, adenoma or focal nodular hyperplasia).

The upper GI Endoscopy revealed grade I esophageal varices and the colonoscopy was normal.

Blood tests showed normal AST, ALT, Bilirubin,

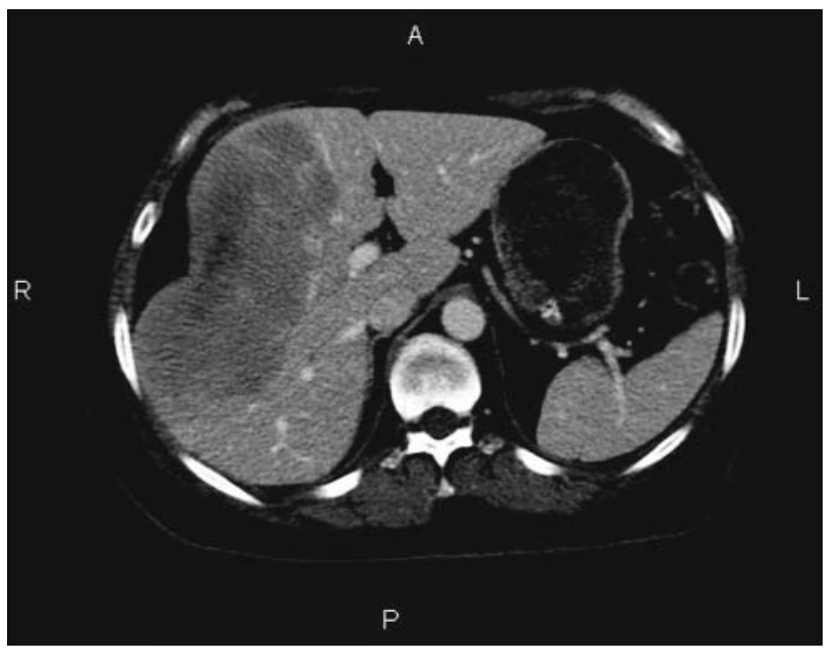

Figure 1 - CT scan revealing a large liver tumor involving segments 4, 5, 6, 8 (volume of segments 2-3: $295 \mathrm{cmc}, 13 \%$ of TLV) 
AFP, CA 19-9 and CEA, and ruled out Hepatitis B or C infection. The only abnormal findings were dyslipidemia and slightly increased levels of CA 15-3 (64.7 U/ml).

Mammography was performed but it did not reveal any pathologic findings.

A multidisciplinary team decided to proceed with laparotomy with intraoperative biopsy and if frozen pathologic examination will reveal a malignant tumor to employ a strategy aiming at the resection of the tumor.

On February $19^{\text {th }}$ 2015, the patient underwent laparotomy. Intraoperative findings (by palpation and ultrasonography) confirmed the presence of the tumor involving segments 4, 5, 6 and 8 with encasement of the middle hepatic vein and intraoperative flash-frozen biopsy established the diagnosis of NHL confined to the liver.

Because the initial complete resection of the tumor was not possible due to the insufficient remnant liver parenchyma (volume of segments 2 and 3 represented only $13 \%$ of TLV), an ALPPS approach was attempted.

The operation started with cholecystectomy, then, it was performed the mobilization of the right hemiliver with ligation and transsection of the accessory veins draining into the inferior vena cava (IVC). The right hepatic vein was identified, but it was not transsected.

Dissection of the hepato-duodenal ligament was carried out, identifying the right hepatic artery, right portal branch and right bile duct. The right portal branch was transsected and the two stumps were sutured, while the right hepatic artery and right bile duct were left intact (fig. 2a). The parenchyma transsection was performed following a plane located $1-2 \mathrm{~cm}$ to the right of the left intersectional plane (fig. $2 b$ ), splitting the liver in two parts (segments 2-3 - on the left side and the right hemiliver plus segment 4 - on the right side). In order to avoid the development of adhesions between the two parts of the liver, an inflated Foley catheter has been placed between them (along the transsection plane - fig. $2 b$ ). Sampling of the lymphnodes located paracaval and in the hepatic pedicle has also been performed.

The postoperative course was uneventful: the patient did not develop either biliary fistula, or ascites (the amount of abdominal drainage fluid was less than $200 \mathrm{ml}$ daily). AST and ALT levels decreased from 255 $\mathrm{U} / \mathrm{I}$ and $282 \mathrm{U} / \mathrm{I}$, respectively (in postoperative day 1POD 1) to $51 \mathrm{U} / \mathrm{l}$ and $77 \mathrm{U} / \mathrm{l}$, respectively in the POD 5. The values of Bilirubin were within normal range every day.

In the POD 11, a CT scan with volumetric evaluation was performed, revealing the absence of postoperative fluid collections and the volume of segments 2-3 was
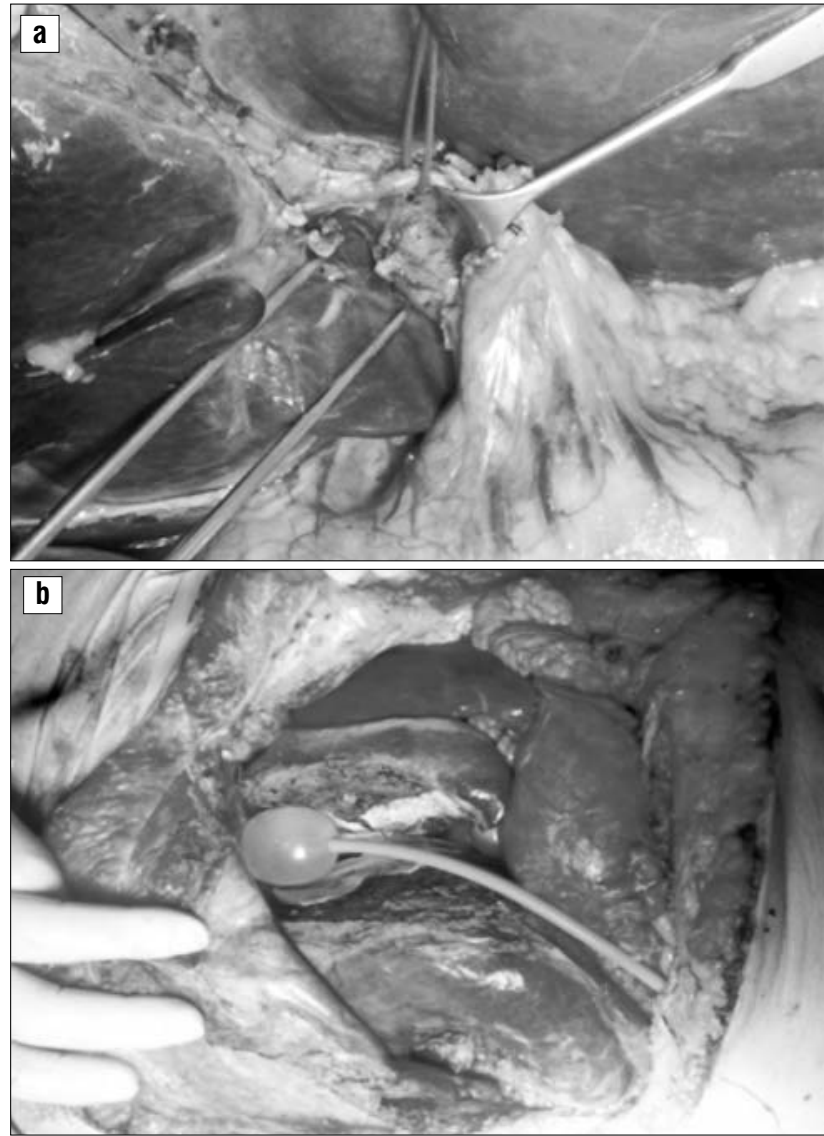

Figure 2 - a. The right portal branch transected and ligated. Right hepatic artery and right bile duct were not ligated. b. A Foley catheter placed on the transected surface of the liver

$586 \mathrm{cmc}$ (fig. 3). Thus, the FLR volume almost doubled after PVL and in situ splitting (from $294 \mathrm{cmc}$ before operation, to $586 \mathrm{cmc}$ after PVL and in-situ splitting), representing $27.3 \%$ of TLV. Thus, it was considered that the second stage could be safely performed, with low risk of postoperative liver failure.

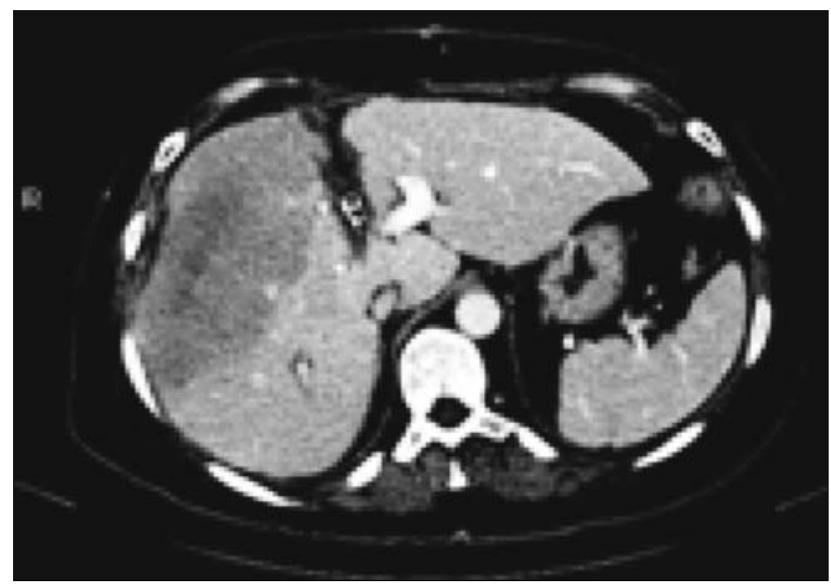

Figure 3 - CT scan performed at 11 days after right portal branch ligation and in-situ splitting (volume of segments 2-3: $586 \mathrm{cmc}$, $27.3 \%$ of TLV) 
On March $9^{\text {th }} 2015$ the patient underwent liver resection (right trisectionectomy). During laparotomy, the right hepatic artery and the right bile duct were ligated and divided, the right and middle hepatic veins were transsected and the specimen was removed and sent for pathologic examination (fig. 4).

Postoperatively the patient presented transient ascites (1100 $\mathrm{ml}$ during the second postoperative day) treated with Manitol, achieving minimal peritoneal drainage by POD 6. AST and ALT levels became normal by the POD 5 and Bilirubin level was normal throughout.

The patient was discharged on POD 9 after the second operation, in good clinical condition, with normal liver enzymes.

The paraffin embedded examination of the tumor revealed a dense lymphoid infiltrate with small-sized cells that formed lymphoepithelial lesions on bile capillaries (Fig 5) and confirmed the diagnosis of NHL of the liver, without malignant cells in the harvested lymph nodes.

Imunohistochemical examination indicate that the lymphoma proliferation was positive for $\mathrm{CD} 20$ (fig. 6) (B-cell marker) and negative for CD3 (T-cell marker), Cyclin D1 (mantle-cell lymphoma marker), CD23 (follicular dendritic cell marker) and BCL6 (follicle centre cell marker). The diagnosis was low-grade small B-cell extranodal marginal zone lymphoma MALT (mucosaassociated lymphoid tissue) type.

Bone marrow trephine biopsy was without lymphoma infiltration.

The patient received 6 cycles of postoperative chemotherapy, with R-CHOP regimen (Rituximab, Cyclophosphamide, Doxorubicin, Vincristine, Prednisone), starting in June 2015.

At the time of writing this manuscript, one year after liver resection, the patient is alive, in good clinical condition, without any evidence of recurrent disease.

\section{DISCUSSION}

To the best of our knowledge this is the first case reported in the literature of PHL resected by ALPPS procedure.

$\mathrm{PHL}$ is a rare pathologic condition, representing $0.016 \%$ of all cases of non-Hodgkin lymphomas (1). Until recently, the treatment of patients with PHL consisted of combined chemotherapy, but the prognosis was dismal in most cases, the median survival being 15.3 months after the diagnosis (according to a review of 72 cases) (8). However, due to the small number of cases, the optimal therapy is still unclear, but in the last years, liver

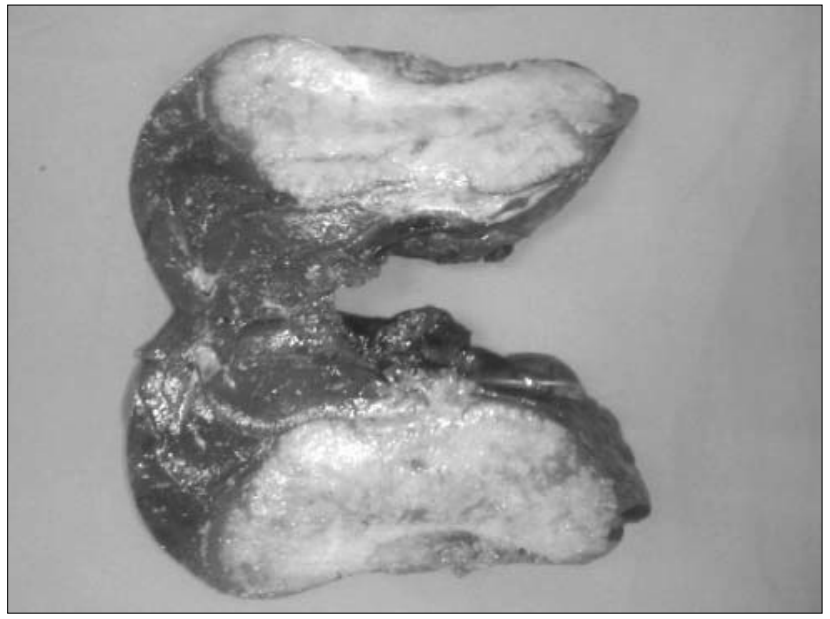

Figure 4 - The resected specimen

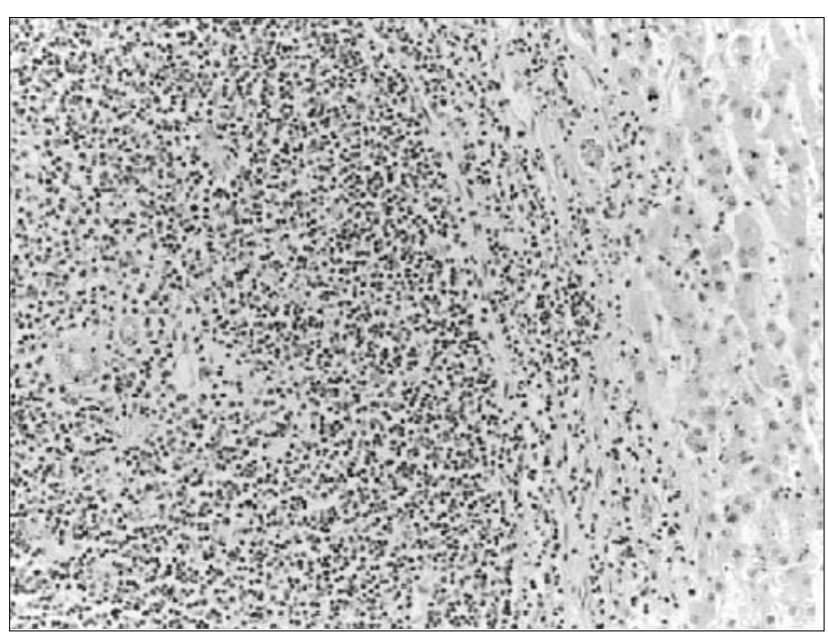

Figure 5 - Liver tumor - small-sized lymphoid proliferation

(HE stain, ob. 20x)

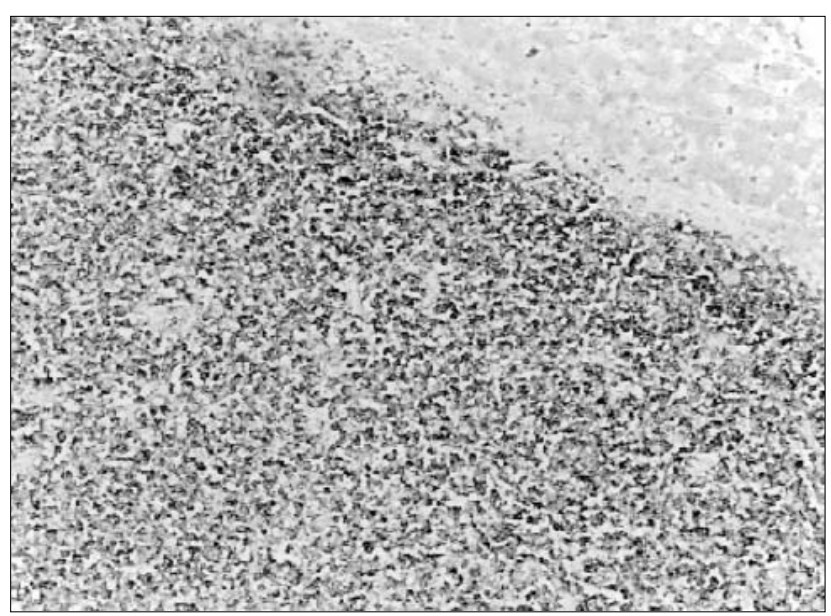

Figure $\mathbf{6}$ - Liver tumor - small B-cell lymphoid proliferation, CD20 positive (IHC stain for CD20, ob. 20x)

resection associated with chemotherapy seems to be the treatment of choice in patients with PHL $(3-7,9,10)$. 
That was the reason why it was decided to resect this large liver tumor in the patient presented above, although initially complete resection was not possible due to the insufficient volume of the FLR.

Over the past two decades, the most used methods to enlarge the volume of FLR in patients with initially unresectable liver tumors were liver resection after PVE/PVL and "two-stage" liver resection (with or without PVE/PVL). The ratio of FLR hypertrophy is calculated with formula: $100 \times$ (FLR Volume after PVE - FLR volume before PVE/FLR volume before PVE) (11). By applying PVE or "two-stage" liver resection to increase the volume of FLR, the ratio of FLR hypertrophy ranged between $20 \%$ and $50 \%$ (12-17) within 2-8 weeks, enabling RO liver resection in almost $66 \%$ of these patients $(11,16,18-21)$. Few years ago, ALPPS has been launched as a novel surgical modality able to achieve a more robust hypertrophy of the FLR in a shorter period of time.

In a previous paper published by our center, the ratio of FLR hypertrophy achieved by PVL or "two-stage" liver resection associated with PVL was $43.8 \%$ at 6 weeks (16). In patient presented above, the ratio of FLR hypertrophy was $98 \%$ at 11 days, significantly higher than those reported with PVL. The higher hypertrophy ratio achieved by ALPPS procedure in the patient presented above is similar to those reported by other authors (2224), who revealed a statistically significant increase in FLR volume after ALPPS than in patients undergoing $\mathrm{PVE} / \mathrm{PVL}$, allowing the performance of $\mathrm{RO}$ resections in almost $100 \%$ of patients subjected to this new approach $(25,26)$. Moreover, recent studies revealed that in patients who failed to achieve sufficient FLR hypertrophy after PVE, the performance of ALPPS was an effective strategy, able to induce a FLR gain which allowed subsequent $\mathrm{RO}$ resection $(26 ; 27)$.

Moreover, this higher hypertrophy after ALPPS was achieved within 6-10 days (22-24), unlike the hypertrophy induced by PVE which was observed after 2-6 weeks. A multicentric analysis revealed an 11-fold faster hypertrophy after ALPPS than after PVE (28). As hypertrophy is achieved more rapidly after ALPPS, the complete resection of the tumor could be performed in a shorter period of time, which offers some benefits: 1 . the risk of disease progression between the two stages of the operation decreases $(25,29) ; 2$. the patient recovers more rapidly, decreasing the length of hospital stay $(25) ; 3$. the postoperative chemotherapy could be delivered sooner than in patients undergoing "twostage" operations (25).

However, the ALPPS procedure presents some drawbacks, the most important being those related to the higher morbidity and mortality rates. Thus, major morbidity (Dindo-Clavien III or more) rates after ALPPS ranged between 27 and $41 \%$ in series presented until now $(24,26,28,30,31)$. Moreover, the high mortality rates (up to $12.5 \%$ in most series) raise concerns about the indications, limits and surgical technique used for ALPPS $(26,28)$. These factors were assessed in some recent papers and the authors recommended avoiding ALPPS in patients older than 60 years-old and in patients with hilar cholangiocarcinoma $(28,32)$. Other experts also recommended avoiding ligation of the right bile duct during the first operation (30) and revealed that obesity, post-stage one biliary fistula and ascites or infected/bilious peritoneal fluid at stage two operation were significantly associated with major morbidity and mortality (26).

The favorable outcome of the patient presented above could be explained by several facts: the patient had normal BMI, was less than 60 year-old, did not receive chemotherapy in the few years leading to the surgical intervention, the tumor was a lymphoma (not a hilar cholangiocarcinoma) and the right bile duct was not ligated during the first stage of the surgical intervention. Furthermore, after the first stage, the patient did not develop biliary fistula or ascites and, at the time of the second operation, infected or bilious peritoneal fluid was not present. Thus, the patient did not present any of the poor prognostic factors reported in the literature, revealing that an accurate indication and an elaborate surgical technique are able to avoid the increased morbidity and mortality rates in patients undergoing ALPPS procedure.

Moreover, the survival of this patient, which is alive and disease-free 1 year after hepatectomy, seems to justify the efforts to resect the PHL. We consider that the disease-free survival of the patient at one year following ALPPS represents a promising result, taking into account the median survival of only 15.3 months reported in a review of the literature which analyzed mainly patients treated with chemotherapy alone (10). In 2010, Yang X. W. presented a series of 9 patients undergoing liver resection and postoperative chemotherapy for $\mathrm{PHL}$ and reported a median survival of 23 months, which seems to be superior to those achieved by chemotherapy alone (6). Moreover, in a more recent series, of 35 patients undergoing liver resection and chemotherapy for PHL, the median survival of patients with B-cell PHL (31.7 months) was statistically significant higher ( $p$ value $<0.05$ ) than those achieved in patients presenting T-cell PHL (22.9 months) (33).

In conclusion, although the optimal treatment of primary hepatic lymphoma is still unclear, liver resec- 
tion associated with chemotherapy seems to prolong survival of these patients. ALPPS could represent an attractive surgical strategy aiming conversion to resectability of initially unresectable liver tumors, when the indication is adequate and surgical technique is well elaborate. PHL seems to be a type of tumor which can benefit from ALPPS procedure, with low postoperative risks. The hypertrophy of the future liver remnant achieved by ALPPS is higher than those reported after portal vein embolization/ligation and it is accomplished in a shorter period. Thus, ALPPS enables resection of initially unresectable liver tumors in most patients with initially insufficient $F L R$, decreasing the risk of tumor progression until the second operation.

\section{REFERENCES}

1. Agmon-Levin N, Berger I, Shtalrid M, Schlanger H, Sthoeger ZM: Primary hepatic lymphoma: a case report and review of the literature. Age Ageing. 2004 Nov;33(6):637-40.

2. Page RD, Romaguera JE, Osborne B, Medeiros LJ, Rodriguez J, North $L$, et al. Primary hepatic lymphoma: favorable outcome after combination chemotherapy. Cancer. 2001 Oct 15;92(8):2023-9.

3. Bouliaris K, Christodoulidis G, Koukoulis G, Mamaloudis I, Ioannou M, Bouronikou $E$, et al. A primary hepatic lymphoma treated with liver resection and chemotherapy. Case Rep Surg 2014;2014:749509.

4. Eidt S, Nebeling M, Pohl C, Siedek M. Neoadjuvant chemotherapy of primary hepatic non-Hodgkin's lymphoma Med Klin (Munich). 2003 Feb 15:98(2):96-9. German

5. Noronha V, Shafi NQ, Obando JA, Kummar S. Primary non-Hodgkin's lymphoma of the liver. Crit Rev Oncol Hematol. 2005 Mar;53(3):199207.

6. Yang XW, Tan WF, Yu WL, Shi S, Wang Y, Zhang YL, et al. Diagnosis and surgical treatment of primary hepatic lymphoma. World $\mathrm{J}$ Gastroenterol. 2010 Dec 21;16(47):6016-9.

7. Zentar A, Tarchouli M, Elkaoui H, Belhamidi MS, Ratbi MB, Bouchentouf SM, et al. Primary hepatic lymphoma. J Gastrointest Cancer. 2014 Sep;45(3):380-2.

8. Kim JH, Kim HY, Kang I, Kim YB, Park CK, Yoo JY, et al. A case of primary hepatic lymphoma with hepatitis $C$ liver cirrhosis. Am J Gastroenterol. 2000 Sep;95(9):2377-80.

9. Lei KI. Primary non-Hodgkin's lymphoma of the liver. Leuk Lymphoma. 1998 Apr;29(3-4):293-9.

10. Avlonitis VS, Linos D. Primary hepatic lymphoma: a review. Eur J Surg. 1999 Aug;165(8):725-9.

11. Abdalla EK, Hicks ME, Vauthey JN: Portal vein embolization: rationale, technique and future prospects. Br J Surg. 2001 Feb;88(2):165-75.

12. Farges 0 , Belghiti J, Kianmanesh $R$, Regimbeau JM, Santoro $R$, Vilgrain V, et al. Portal vein embolization before right hepatectomy: prospective clinical trial. Ann Surg. 2003 Feb;237(2):208-17.

13. Jaeck D, Bachellier P, Nakano H, Oussoultzoglou E, Weber JC, Wolf $P$, et al. One or two-stage hepatectomy combined with portal vein embolization for initially nonresectable colorectal liver metastases. Am J Surg. 2003 Mar;185(3):221-9.

14. Ko GY, Sung KB, Yoon HK, Kim JH, Weon YC, Song HY. Preoperative portal vein embolization with a new liquid embolic agent. Radiology. 2003 May;227(2):407-13. Epub 2003 Mar 13.

15. Tanaka K, Shimada H, Matsuo K, Ueda M, Endo I, Togo S. Remnant liver regeneration after two-stage hepatectomy for multiple bilobar colorectal metastases. Eur J Surg Oncol. 2007 Apr;33(3):329-35. Epub 2006 Nov 30.
16. Popescu I, Alexandrescu S, Croitoru A, Boros M: Strategies to convert to resectability the initially unresectable colorectal liver metastases. Hepatogastroenterology. 2009 May-Jun;56(91-92):739-44.

17. Azoulay D, Castaing D, Smail A, Adam R, Cailliez V, Laurent A, et al. Resection of nonresectable liver metastases from colorectal cancer after percutaneous portal vein embolization. Ann Surg. 2000 Apr; 231(4):480-6.

18. Adam R, Laurent A, Azoulay D, Castaing D, Bismuth H. Two-stage hepatectomy: A planned strategy to treat irresectable liver tumors. Ann Surg. 2000 Dec;232(6):777-85.

19. Jaeck D, Oussoultzoglou E, Rosso E, Greget M, Weber JC, Bachellier P. A two-stage hepatectomy procedure combined with portal vein embolization to achieve curative resection for initially unresectable multiple and bilobar colorectal liver metastases. Ann Surg. 2004 Dec;240(6):1037-49; discussion 1049-51.

20. Popescu I, David L, Brasoveanu V, Boros M, Hrehoret D. Two-stage hepatectomy: an analysis of a single center's experience. Magy Seb. 2006 Jun;59(3):184-9.

21. Wicherts DA, Miller R, de Haas RJ, Bitsakou G, Vibert E, Veilhan LA, et al. Long-term results of two-stage hepatectomy for irresectable colorectal cancer liver metastases. Ann Surg. 2008 Dec;248(6):9941005.

22. Alvarez FA, Iniesta J, Lastiri J, Ulla M, Bonadeo Lassalle F, de Santibañes E. New method of hepatic regeneration. Cir Esp. 2011 Dec;89(10):645-9. Spanish

23. de Santibañes E, Alvarez FA, Ardiles V. How to avoid postoperative liver failure: a novel method. World J Surg. 2012 Jan;36(1):125-8.

24. Schnitzbauer AA, Lang SA, Goessmann H, Nadalin S, Baumgart J, Farkas SA, et al. Right portal vein ligation combined with in situ splitting induces rapid left lateral liver lobe hypertrophy enabling 2staged extended right hepatic resection in small-for-size settings. Ann Surg. 2012 Mar;255(3):405-14.

25. Tanaka K, Matsuo K, Murakami T, Kawaguchi D, Hiroshima Y, Koda K, et al. Associating liver partition and portal vein ligation for staged hepatectomy (ALPPS): short-term outcome, functional changes in the future liver remnant, and tumor growth activity. Eur J Surg Oncol. 2015 Apr;41(4):506-12.

26. Truant S, Scatton 0, Dokmak S, Regimbeau JM, Lucidi V, Laurent A, et al. Associating liver partition and portal vein ligation for staged hepatectomy (ALPPS): impact of the inter-stages course on morbimortality and implications for management. Eur J Surg Oncol. 2015 May;41(5):674-82.

27. Tschuor C, Croome KP, Sergeant G, Cano V, Schadde E, Ardiles V, et al. Salvage parenchymal liver transection for patients with insufficient volume increase after portal vein occlusion -- an extension of the ALPPS approach. Eur J Surg Oncol. 2013 Nov;39(11):1230-5.

28. Schadde E, Ardiles V, Slankamenac K, Tschuor C, Sergeant G, Amacker N, et al. ALPPS offers a better chance of complete resection in patients with primarily unresectable liver tumors compared with conventional-staged hepatectomies: results of a multicenter analysis. World J Surg. 2014 Jun;38(6):1510-9.

29. de Santibañes E, Clavien PA. Playing Play-Doh to prevent postoperative liver failure: the "ALPPS" approach. Ann Surg. 2012 Mar;255(3): 415-7.

30. Dokmak S, Belghiti J. Which limits to the "ALPPS" approach? Ann Surg. 2012 Sep;256(3):e6; author reply e16-7.

31. Nadalin S, Capobianco I, Li J, Girotti P, Konigsrainer I, Konigsrainer A. Indications and limits for associating liver partition and portal vein ligation for staged hepatectomy (ALPPS). Lessons Learned from 15 cases at a single centre. Z Gastroenterol. 2014 Jan;52(1):35-42.

32. Li J, Girotti P, Konigsrainer I, Ladurner R, Konigsrainer A, Nadalin S. ALPPS in right trisectionectomy: a safe procedure to avoid post-operative liver failure? J Gastrointest Surg. 2013 May;17(5): 956-61.

33. Zhao Q, Liu HP, Gu YJ, Cong WM. Clinicopathological and survival features of primary hepatic lymphoma: an analysis of 35 cases. Zhonghua Zhong Liu Za Zhi. 2013 Sep;35(9):689-92. Chinese 